\title{
Agreement with Microcurrent Conductance, Serum Myoglobin, and Diagnostic Ultrasound When Evaluating Delayed Onset Muscle Soreness
}

\author{
Timothy Hui ${ }^{1}$, Jerrold Petrofsky ${ }^{1}$, Michael Laymon ${ }^{2}$ \\ ${ }^{1}$ Loma Linda University, Loma Linda, USA \\ ${ }^{2}$ Touro University, Henderson, USA \\ Email: timothyehui@gmail.com
}

Received 27 February 2014; revised 20 March 2014; accepted 18 April 2014

Copyright (C) 2014 by authors and Scientific Research Publishing Inc.

This work is licensed under the Creative Commons Attribution International License (CC BY). http://creativecommons.org/licenses/by/4.0/

(c) (i) Open Access

\begin{abstract}
Purpose/Hypothesis: To determine if agreement exists between microcurrent conductance through the skin over an injury, myoglobin levels in the serum, and diagnostic ultrasound measurements of swelling. Number of Subjects: 140. Materials/Methods: Subject underwent microcurrent conductance, serum myoglobin, diagnostic ultrasound, and strength testing before and after delayed onset muscle soreness (DOMS) was induced. Non-control subjects were also given a therapy wrap, dry heat, moist heat, or cold, either applied immediately or delayed 24 hours. Results: After induction of DOMS, there was agreement between significant $37 \%$ decreases in microcurrent conductance, significant $37 \%$ serum myoglobin increases, and significant $20 \%$ increases in quadriceps size, measured by diagnostic ultrasound. When either dry heat or cold was applied immediately, changes in these measurements were insignificant and less than $10 \%$. Agreement was weaker when treatment was delayed 24 hours. Also, cold packs showed less than a $5 \%$ decrease in microcurrent conductance for covered areas compared to a $22 \%$ decrease for uncovered areas around the knee with 24 hour delay. Moist heat packs showed an insignificant change overall, but a significant $11 \%$ decrease for the covered center of the quadriceps with immediate treatment. Strength decreased 25\% after DOMS, and all immediate treatments, along with 24 hour delayed cold and moist heat showed significantly smaller decreases. Conclusions: Changes in microcurrent conductance through the skin over injured tissue appear to be indicative of initial injury and recovery, and also for detecting the specific area of the injury. Clinical Relevance: Microcurrent through the skin over injured tissue appears to be a promising objective measurement of tissue injury as well as recovery from injury.
\end{abstract}

\section{Keywords}

Soft Tissue Injuries, Electric Resistance, Outcome Assessment, Ultrasonography 


\section{Introduction}

Heat and cold modalities have been used to treat musculoskeletal injuries for years. Some studies have reported positive results from these therapies, but these studies have generally relied on subjective outcome measures [1][3]. Purely objective outcomes, such as blood tests, have been attempted, but results have been mixed [4] [5]. However, microcurrent conductance has shown promise as an effective, non-invasive outcome measure of musculoskeletal injury [6].

One benefit of evaluating soft tissue injury with microcurrent conductance is that it does not require any subjective input from the patient. Therefore, differences in pain perception, which can be significant, are eliminated [7].

Research has shown a 70\% decrease in microcurrent conductance in the skin over an injury compared to uninjured tissue [6]. The proposed reason for this decrease is that tissue conductance decreases with hypoxic damage [8]. Wounds demonstrate hypoxia from increased metabolic activity [9], so the decrease in conductance could be due to microcurrent travelling through hypoxic tissue. This was tested by inducing hypoxia for five minutes, with results showing significant decreases in microcurrent [6].

Although more invasive than microcurrent, blood tests are another objective measure of body function. A blood test that could detect muscular injury is serum myoglobin. Since myoglobin is normally only within muscle cells, its presence in the blood indicates disruption of the sarcolemma of those cells [10]. As swelling occurs concurrently with this disruption, the size of the muscle should increase and be visible with diagnostic ultrasound imaging. Studies have shown increases in serum myoglobin occurring alongside delayed onset muscle soreness (DOMS) [10] [11]. In one study, serum myoglobin levels rose in individuals undergoing a therapeutic exercise program, with levels significantly higher on the third day after exercise [11]. In another exercise study, untrained subjects experiencing DOMS after exercise also showed significant increases in myoglobin. Also, once subjects had undergone a regiment of physical training, they experienced less DOMS and had significantly lower myoglobin levels than at the start [12]. For more substantial muscular injury, a case study for exercise induced rhabdomyolysis, when extreme overuse results in muscle breakdown, blood tests showed a greater than 50 fold increase in myoglobin, as well as increased creatine kinase and aspartate aminotransferase [13]. However, studies have also shown a great variation in levels of myoglobin within the blood. This is due to the fact that blood proteins are diluted throughout the subject's blood supply, and that kidney filtration is constantly removing aberrant particles [14] [15].

One commonly used therapy for soreness is heat therapy with the moist heat pack being the most common form of this therapy [16]. The physiological effects of heat therapy have been well studied. Heat therapy increases circulation when therapy is applied between 30 and 45 degrees Celsius, which is the likely cause of its therapeutic effects [17].

Research has shown heat to be effective in reducing pain [18] [19], but functional outcomes give conflicting results. When testing subjects with arthrogenic inhibition for muscles around the knee, a moist heat pack did not create any significant changes in the strength of knee extension [18]. However, another study showed strength increases at the knee after heat for subjects with osteoarthritis [19].

Another common modality for muscle soreness is cold therapy. The mechanism of cold therapy, or cryotherapy, has not been as well studied as heat. However, cryotherapy has been shown to significantly reduce pain in subjects with muscle damage from exercise [20]. Though the physiological mechanisms are not known, cryotherapy is commonly used for acute soft tissue injuries to reduce pain and swelling. It may also have an anti-inflammatory effect [3].

With the heat or cold therapy, it is possible that there would be less injury shown either through measurements of serum myoglobin or through microcurrent conductance, and also less swelling as viewed by diagnostic ultrasound. It is also possible that a function change, such as muscle strength, would also be affected by the quantity of injury. Previous studies have shown microcurrent and myoglobin changes with soft tissue injury, along with functional changes. However, the outcomes have not been tested together and compared for agreement. Ideally, there should be agreement, but due to the limitations listed above, weaker agreement should be observed in less ideal situations. Therefore, the purpose of this study was to determine if agreement exists between microcurrent resistance through the skin over an injury, myoglobin levels in the serum, and swelling as determined by diagnostic ultrasound. Also, we wished to determine if there was a corresponding functional change. 


\section{Subjects}

This study recruited 140 healthy subjects, between age 20 - 40, and free of any orthopedic or neurological conditions, and between the ages of 20 and 40. Subjects were randomly divided into 1 control and 6 treatment groups: immediate and 24 hour delayed cold, immediate and 24 hour delayed heat, and immediate and 24 hour delayed moist heat. The ages, heights and weights of the groups showed no statistical difference.

The general characteristics are listed in Tables 1-7. Subjects were recruited from the students and faculty of Loma Linda University, or from the community. The experimental protocol, approved by the Institutional

Table 1. Demographics of control group.

\begin{tabular}{ccccc}
\hline & age (years) & height $(\mathbf{c m})$ & weight $(\mathbf{k g})$ & BMI \\
\hline mean & 25.3 & 165.9 & 63.7 & 23.1 \\
sd & 3.0 & 6.0 & 10.4 & 3.5 \\
\hline
\end{tabular}

Table 2. Demographics—cold immediate.

\begin{tabular}{ccccc}
\hline & age (years) & height $(\mathbf{c m})$ & weight $\mathbf{( k g )}$ & BMI \\
\hline mean & 25.5 & 174.4 & 67.2 & 22.0 \\
sd & 2.7 & 9.2 & 12.4 & 2.5 \\
\hline
\end{tabular}

Table 3. Demographics-24 hour delayed cold.

\begin{tabular}{ccccc}
\hline & age (years) & height $(\mathbf{c m})$ & weight $(\mathbf{k g})$ & BMI \\
\hline Mean & 26.1 & 170.3 & 74.1 & 25.3 \\
Sd & 2.8 & 8.6 & 26.6 & 7.7 \\
\hline
\end{tabular}

Table 4. Demographics_-dry heat immediate.

\begin{tabular}{ccccc}
\hline & age (years) & height $(\mathbf{c m})$ & weight $(\mathbf{k g})$ & BMI \\
\hline Mean & 26.1 & 166.1 & 67.0 & 24.2 \\
Sd & 2.6 & 10.1 & 12.6 & 3.5 \\
\hline
\end{tabular}

Table 5. Demographics—-24 hour delayed dry heat.

\begin{tabular}{ccccc}
\hline & age (years) & height $\mathbf{( c m )}$ & weight $\mathbf{( k g )}$ & BMI \\
\hline Mean & 24.9 & 166.3 & 62.6 & 22.5 \\
Sd & 3.0 & 10.6 & 15.5 & 4.0 \\
\hline
\end{tabular}

Table 6. Demographics-moist heat immediate.

\begin{tabular}{ccccc}
\hline & age (years) & height $(\mathbf{c m})$ & weight $\mathbf{( k g )}$ & BMI \\
\hline Mean & 24.9 & 172.8 & 69.0 & 23.0 \\
Sd & 1.9 & 7.6 & 9.7 & 2.2 \\
\hline
\end{tabular}

Table 7. Demographics—24 hour delayed moist heat.

\begin{tabular}{rcccc} 
& age (years) & height $(\mathbf{c m})$ & weight $(\mathbf{k g})$ & BMI \\
\hline Mean & 26.3 & 167.4 & 66.7 & 23.6 \\
Sd & 2.8 & 7.6 & 13.7 & 3.4 \\
\hline
\end{tabular}


Review Board of Loma Linda University, was explained to each subject and the subjects gave their written inconsent for the study.

\section{Methods}

Subjects were tested over five consecutive days, generally Monday to Friday. On day 1, baselines were established for all outcome measures. On day 1, 3, 4, and 5, microcurrent and strength measurements were taken. Blood was drawn on days 1 and 4 . Subjects underwent strenuous exercise on day 2 to create DOMS.

A prototype device from Mettler Electronics (Anaheim, CA) called a Zone Finder measured microcurrent in microamperes by running a calibrated 100 microamp DC microcurrent between two probes. Distance between probes was kept constant at $2.5 \mathrm{~cm}$ for all measurements. The force on each probe was measured with force gauges and kept constant at 3.6 newtons for all measurements. The subject's skin was cleaned with alcohol to remove oil and dirt and readings were taken after cotton pads soaked in $0.9 \%$ saline were placed into the probes. By reporting microamperes, this instrument measures conductance, which is the reciprocal of electrical resistance $(\mathrm{C}=1 / \mathrm{R})$.

Plasma myoglobin was tested using commercially available enzyme-linked immunosorbent assay (ELISA) kits in a 96 well plate format according to the manufacturers' instructions-Plasma Myoglobin (MG017C: CalBiotech, Spring Valley, CA)

Musculoskeletal ultrasound (US), a useful imaging tool in examining muscle damage, was imaged with a L14-6 MHz linear array transducer (Mindray-M7 Diagnostic Ultrasound System) and acoustic coupling gel, and aligned vertically with the quadriceps muscle belly. The spot of measurement was $40 \%$ of the distance between the crest of the hip and top of the patella and marked, and with the femur in the center of view. Longitudinal and cross sectional views were both taken.

Quadriceps strength was tested on seated subjects with a dynometer. Subjects were told to extend their knee in an isometric contraction with maximum force, and hold their force constant for 3 - 4 seconds.

Subjects in test groups received heat therapy using an 8 hour heatwrap from ThermaCare( ${ }^{\circledR}$ ) (Pfizer Consumer Healthcare, Richmond, VA), a two hour moist heat pack, or a 20 minute cold wrap. The dry heat, moist heat, and cold wraps all came in a standardized size, large enough to surround the quadriceps. However, the moist heat wraps were half the size of the other wraps, leaving more of the distal and proximal quadriceps uncovered.

\section{Procedures}

On measurement days, blood samples were first taken if indicated for the day. Subjects were evaluated then for microcurrent and strength. For microcurrent, measurements were taken at the distal, mid, and proximal quadriceps, medially, centrally, and laterally while supine. A single measurement at the midpoint of the quadriceps was also taken while seated, making a total of ten measurements per leg per day. The unit was calibrated to 100 microamps before each subject was tested.

On the exercise day, subjects were exercised with three 5-minute sets of squats with no added resistance. During those 5 minutes, subjects were told to perform as many squats as possible, with three minutes to rest between sets. Heat or cold was applied after exercise or the following day, as indicated by group.

\section{Statistical Analysis}

P-values were generated using a two-tailed t-test assuming equal variances. Results were normalized into percent change for results.

\section{Results}

Figure 1 shows the average microcurrent changes from baseline for all groups. For microcurrent, decreases were significant $(\mathrm{p}<0.01)$ from baseline after DOMS was induced. In the treatment groups, there were significant changes from control after exercise for immediate heat and 24 hour delayed heat $(\mathrm{p}<0.05)$. Looking at specific areas, the greatest changes for the control were seen around the knee, where there was a $43 \%$ decrease after three days as opposed to a $28 \%$ decrease for all other areas. Of the other areas, the greatest change was at the center of the quadriceps while supine, with a $37 \%$ decrease. With immediate heat, the changes were significantly less than control with a $5 \%$ decrease $(\mathrm{p}<0.001)$ around the knee on the third day, while the other areas 


\section{Percentage of Microcurrent Conductance Baseline after DOMS}

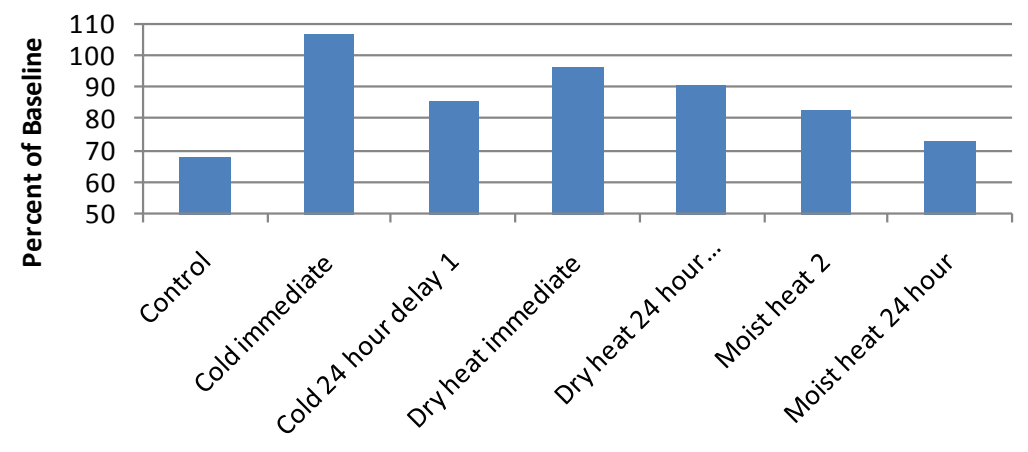

Figure 1. Average microcurrent changes in measurements from both quadriceps selected from the day with a greater decrease of the final two days. Cold immediate, Dry heat immediate, and Dry heat 24 hour delayed were significantly different from Control $(\mathrm{P}<0.05)$. 1. Excluding knee areas, Cold 24 hour delay was $95 \%$ of baseline, $\mathrm{P}<0.05$. 2. Focusing on bilateral midpoint readings, Moist heat was $89 \%$ of baseline, $\mathrm{P}<0.05$.

showed a $3 \%$ decrease $(\mathrm{p}<0.00001)$. When heat was delayed 24 hours, changes were significantly less $(\mathrm{p}<$ $0.001)$ at the knee (43\% decrease for control, $9 \%$ decrease for 24 hour heat) and at all other areas (28\% decrease for control, $9 \%$ decrease for 24 hour heat).

For immediate moist heat, changes were insignificant. However, taking only the 4 readings from the middle of the quadriceps, which was definitely covered by the pad, changes were significantly different from control (P $<0.01$ ) with an $11 \%$ decrease. When moist heat was delayed 24 hours, changes were insignificant, with the knee decreased $34 \%$ while the other areas decreased $24 \%$.

When utilizing immediate cold therapy, decreases in microcurrent were significantly different than control for all days tested, though knee levels were consistently more decreased than the other areas. When cold was delayed for 24 hours, changes were insignificant when taken as a whole, but different effects were seen depending on the specific area on the quadriceps. For areas other than the knee, a significant change occurred $(\mathrm{P}<0.05)$, with decreases from $28 \%$ on the first day to only a less than $2 \%$ decrease on the last day. Knee decreases were insignificant, going from $43 \%$ on the first day to $22 \%$ on the last day. Treatment effect was the least for the lateral knee, where decreases went from $45 \%$ the first day to $29 \%$ the final day.

Myoglobin levels significantly increased after induced DOMS when no treatment was applied, as well as when any treatment was delayed for 24 hours. However, no matter what treatment was applied immediately after treatment, the results were essentially the same, with no significant change. When measured by Ultrasound, quadriceps size significantly increased $(\mathrm{p}<0.01)$ by $20 \%$ in the control group three days after induced DOMS.

Figure 2 shows the change in myoglobin in the serum after exercise. The average myoglobin pre exercise was $33.0+/-4.6$ micrograms per liter of blood. The largest increases in myoglobin were after dry, moist and cold at 24 hours. These changes in myoglobin (\% above rest in Figure 2) were significantly higher than baseline but not different form each other. ( $p>0.05)$ Heat and cold immediately did allow for an increase in myoglobin but it was not significantly different than the resting data $(\mathrm{p}>0.05)$. From the diagnostic ultrasound date, quadriceps size increased significantly compared to resting $(\mathrm{p}<0.01)$. For the treatment groups, increases in quadriceps size were not significantly different than baseline.

Figure 3 shows the average strength changes from baseline for all groups.For strength, all of the treatment groups demonstrated smaller declines after DOMS compared to the control. The dry and moist heat groups showed the least strength decline. Changes for all groups, except dry heat 24 hour delayed, demonstrated significant changes $(\mathrm{p}<0.05)$.

\section{Discussion}

In the past, studies have shown changes from various outcome measures as a result of tissue injury [1] [4]. Mi- 


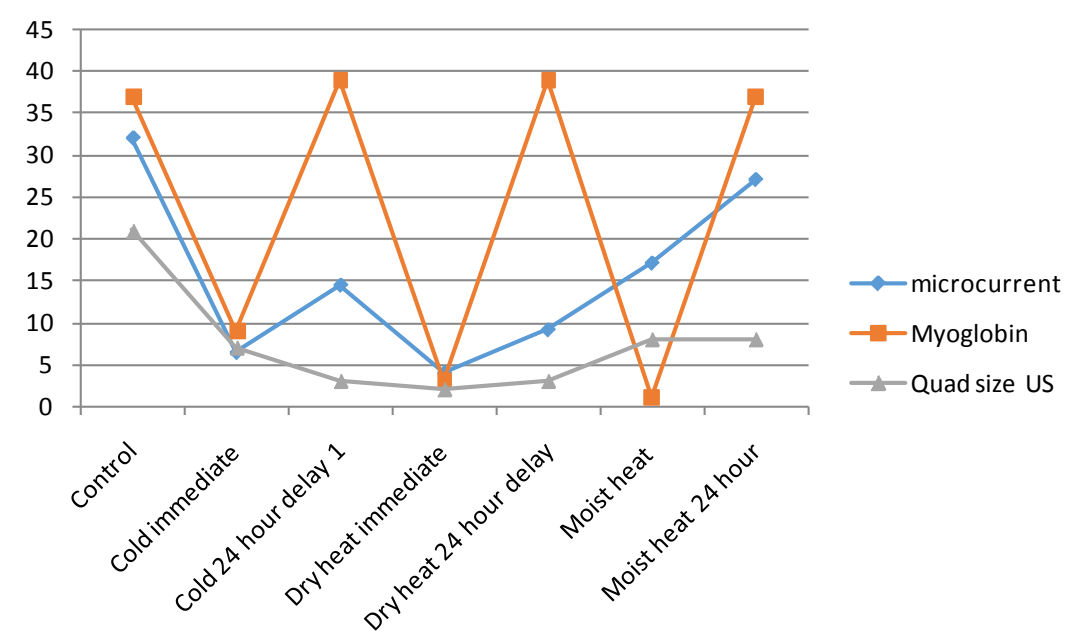

Figure 2. Absolute value of percentage change in microcurrent, myoglobin, and quadriceps size measured by ultrasound in the control and heat, moist heat and control groups. Each group represents the average data on 20 subjects recorded 2 or 3 days after DOMS induction.

\section{Percentage of Strength Baseline after DOMS}

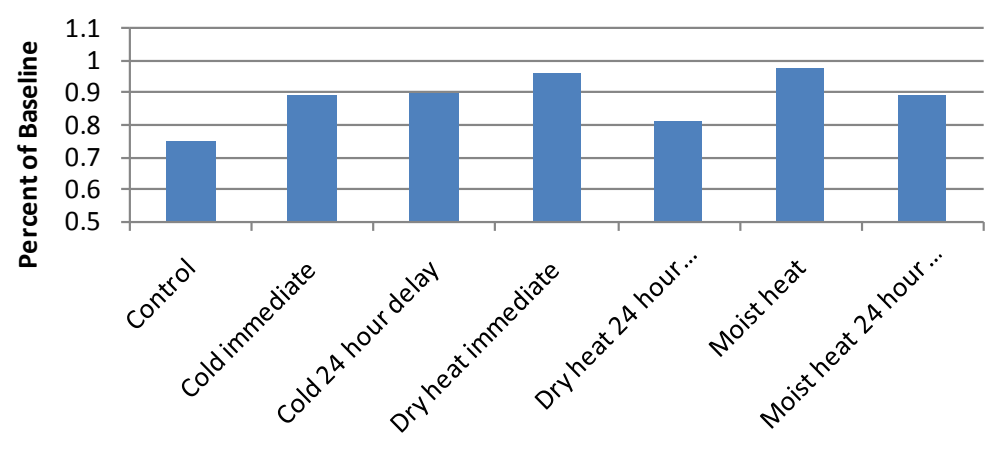

Figure 3. Strength measurements from the day with a greater decrease of the final two days. All groups, except Dry heat 24 hour delayed, were significantly different from control $(\mathrm{p}<0.05)$.

crocurrent conductance, serum myoglobin, and muscular strength have shown changes as a result of tissue injury [6] [10] [18]. However, these outcome measures have not been tested concurrently so their result could be compared. In this study, we tested and compared microcurrent conductance, serum myoglobin, muscular strength, and also swelling with diagnostic ultrasound on subjects where delayed onset muscle soreness was induced, and also where heat or cold therapy was applied after injury.

Microcurrent readings showed significant decreases after DOMS was induced, creating a strong inverse relationship with myoglobin levels, which rose significantly. Microcurrent decreases did not occur when any of the three treatments were immediately applied, where microcurrent showed slight decreases the following day, but conductance was essentially unchanged from baseline on later days. Myoglobin, as well, showed little change with immediate treatment, thus showing high agreement with microcurrent. Therefore, in terms of DOMS effects, serum myoglobin and microcurrent conductance show strong agreement.

Comparing microcurrent and myoglobin with ultrasound, strong agreement was observed in the control group and with immediate dry heat and cold. Microcurrent and Ultrasound show strong agreement except for moist heat delayed 24 hours.

Changes for specific areas showed differences between heat and cold. These were likely due to the physio- 
logical differences between heat and cold therapy. Heat therapy increases circulation. Thus, areas surrounding but not covered by the larger dry heat pack, such as the knee, would receive similar benefits as areas under the pad. However, cold therapy does not increase circulation. Therefore, areas not covered by the pad, such as the knee, would receive less benefit from the therapy. This change is especially pronounced with 24 hour delayed cold, where the delay would allow for more damage to occur. The lateral knee, which was definitely not covered, had far less of a treatment effect (45\% decrease to $29 \%$ ) compared to non-knee areas (28\% decrease to $2 \%$ ). These results suggest that microcurrent conductance can be used to determine injury and recovery not only for an injury, but also the specific location of tissues that have been injured. The knee likely had more damage as more movement and forces are transmitted through it, but that does not explain such as large discrepancy.

Another item to note is that the specific proximity to the cold pad for the knee spots was not controlled. The length of subject's thighs varied with their height, but cold pads were all the same size. If cold pads were closer to the knee on shorter subjects, it is possible that they could still receive a treatment effect compared to taller subjects. Despite this, the uncovered area demonstrated much less of a treatment effect compared to the covered area for both cold therapy groups. This may also explain why the moist heat wrap tests were not statistically significant as a whole, but statistically significant at the center of the quadriceps which was definitely covered by the wrap. The moist heat wraps were about half the width of the cold packs and the dry heat packs, so even though the overall numbers showed a smaller decrease than the control, they may not have been able to generate enough of a treatment effect for statistical significance and may also explain the difference observed between microcurrent and ultrasound.

With delayed treatment, myoglobin levels showed little change from the increased levels found in the control group regardless of treatment type. Microcurrent showed a minor decrease with dry heat and cold, and a decrease equal to the control group for moist heat. This may be due to the rate of serum clearance of myoglobin.

Strength changes show strong agreement with ultrasound, microcurrent, and myoglobin levels for all three immediate treatments, with the result being very little change from baseline levels for the two heat therapies, and only $10 \%$ for cold. For delayed treatment, there is less agreement when comparing myoglobin and strength that being a change similar to control for dry heat, though not for moist heat or cold. Also, there is less agreement with microcurrent conductance, where there was a decrease, but to a lesser degree, for cold. It is also important to note that strength changes for the moist heat groups were minimal while microcurrent conductance and myoglobin showed decreases, especially for the delayed moist heat group. This is not surprising since the mid-quad area contains more contractile tissue compared to the knee, which has a much higher percentage of non-contractile tendons. Since the area that generated the strength showed less damage, strength should not be affected. If the tendons experienced severe damage, there would likely be a strength decrease, but DOMS inducing exercise would be less likely to create this sort of tendinous damage. Because of these two reasons, strength would not likely be affected when minor tissue damage is detected.

Since strength decreased along with the injury, another possible avenue of future research is to determine if strength and other movement deficits may be a result of minor soft tissue injury as detected by microcurrent conductance. Another possible future avenue is combining microcurrent, strength, and pain scales, since they have been used in the past, as a method for evaluating the effectiveness for virtually any treatment.

\section{Conclusion}

In conclusion, given the agreement with serum myoglobin and diagnostic ultrasound, it appears that there is evidence that microcurrent conductance is a valid evaluation of tissue injury. It also shows promise as a measurement of identification of the specific location of an injury as well as its recovery, and also for possible function changes as a result of tissue injury. As expected, greater agreement was observed with immediate treatment compared to the less-ideal situation of delayed treatment. Further testing is still required, but results are promising.

\section{Limitations of This Study Were:}

1) Subjects may not have been completely healthy at the time of baseline testing.

2) Fitness level varies for subjects

3) Strength testing required maximal effort from subjects, and this cannot be controlled.

4) Subjects performed as many squats as possible, the number of which varied according to fitness level. 
5) Dirt and oil on the skin affects microcurrent measurements, and though cleaning occurred, error is impossible to eliminate.

6) Myoglobin was not tested every day due to cost and invasiveness of sample acquisition.

\section{Acknowledgements}

This work was supported under a contract from Pfizer Pharmaceuticals, WI173615.

\section{References}

[1] Nadler, S.F., Steiner, D.J., Erasala, G.N., Hengehold, D.A., Abeln, S.B. and Weingand, K.W. (2003) Continuous Low-Level Heatwrap Therapy for Treating Acute Nonspecific Low Back Pain. Archives of Physical Medicine and Rehabilitation, 84, 329-334. http://dx.doi.org/10.1053/apmr.2003.50102

[2] Nadler, S.F., Steiner, D.J., Petty, S.R., Erasala, G.N., Hengehold, D.A. and Weingand, K.W. (2003) Overnight Use of Continuous Low-Level Heatwrap Therapy for Relief of Low Back Pain. Archives of Physical Medicine and Rehabilitationl, 84, 335-342. http://dx.doi.org/10.1053/apmr.2003.50103

[3] Bleakley, C., McDonough, S., Gardner, E., Baxter, G.D., Hopkins, J.T. and Davison, G.W. (2012) Cold-Water Immersion (Cryotherapy) for Preventing and Treating Muscle Soreness after Exercise. Cochrane Database of Systematic Reviews, 2, Article ID: CD008262. http://dx.doi.org/10.1002/14651858.CD008262.pub2

[4] Warren, G.L., Lowe, D.A. and Armstrong, R.B. (1999) Measurement Tools Used in the Study of Eccentric Contraction-Induced Injury. Sports Medicine, 27, 43-59. http://dx.doi.org/10.2165/00007256-199927010-00004

[5] Clarkson, P.M. and Ebbeling, C. (1988) Investigation of Serum Creatine Kinase Variability after Muscle-Damaging Exercise. Clinical Science, 75, 257-261.

[6] Hui, T. and Petrofsky, J. (2013) Injury and Inflammation Detection by the Application of Microcurrent through the Skin. Physical Therapy Rehabilitation Science, 2, 31-38.

[7] Dixon, J.S. and Bird, H.A. (1981) Reproducibility along a $10 \mathrm{~cm}$ Vertical Visual Analogue Scale. Annals of the Rheumatic Diseases, 40, 87-89.

[8] Yasuda, Y., Maruyama, T., Nakamura, H., Arita, T., Ueda, N. and Harada, M. (2006) Pathophysiological Basis for Monitoring of Whole Heart Conductance by 2-Lead System. Circulation Journal, 70, 495-501. http://dx.doi.org/10.1253/circj.70.495

[9] Murdoch, C., Muthana, M. and Lewis, C.E. (2005) Hypoxia Regulates Macrophage Functions in Inflammation. The Journal of Immunology, 175, 6257-6263. http://dx.doi.org/10.4049/jimmunol.175.10.6257

[10] Hirose, L., Nosaka, K., Newton, M., Laveder, A., Kano, M., Peake, J., et al. (2004) Changes in Inflammatory Mediators Following Eccentric Exercise of the Elbow Flexors. Exercise Immunology Review, 10, 75-90.

[11] Al-Nakhli, H.H., Petrofsky, J.S., Laymon, M.S., Arai, D., Holland, K. and Berk, L.S. (2012) The Use of Thermal Infrared Imaging to Assess the Efficacy of a Therapeutic Exercise Program in Individuals with Diabetes. Diabetes Technology \& Therapeutics, 14, 159-167. http://dx.doi.org/10.1089/dia.2011.0187

[12] Croisier, J.L., Camus, G., Venneman, I., Deby-Dupont, G., Juchmès-Ferir, A., Lamy, M., Crielaard, J.M, Deby, C. and Duchateau, J. (1999) Effects of training on exercise-induced muscle damage and interleukin 6 production. Muscle \& Nerve, 22, 208-212. http://dx.doi.org/10.1002/(SICI)1097-4598(199902)22:2<208::AID-MUS8>3.0.CO;2-B

[13] Inklebarger, J., Galanis, N., Kirkos, J. and Kapetanos, G. (2010) Exercise-Induced Rhabdomyolysis from Stationary Biking: A Case Report. Hippokratia, 14, 279-280.

[14] Warren, G.L., Lowe, D.A. and Armstrong, R.B. (1999) Measurement Tools Used in the Study of Eccentric Contraction-Induced Injury. Sports Medicine, 27, 43-59. http://dx.doi.org/10.2165/00007256-199927010-00004

[15] Clarkson, P.M. and Ebbeling, C. (1988) Investigation of Serum Creatine Kinase Variability after Muscle-Damaging Exercise. Clinical Science, 75, 257-261.

[16] Hassan, E.S. (2011) Thermal Therapy and Delayed Onset Muscle Soreness. The Journal of Sports Medicine and Physical Fitness, 51, 249-254.

[17] Andersen, L.L., Jay, K., Andersen, C.H., Jakobsen, M.D., Sundstrup, E., Topp, R. and Behm, D.G. (2013) Acute Effects of Massage or Active Exercise in Relieving Muscle Soreness: Randomized Controlled Trial. Journal of Strength and Conditioning Research, 27, 3352-3359. http://dx.doi.org/10.1519/JSC.0b013e3182908610

[18] Warner, B., Kim, K.M., Hart, J.M. and Saliba, S. (2012) Superficial Heat to the Knee Does Not Affect Quadriceps Function in Individuals with Quadriceps Inhibition. Journal of Sport Rehabilitation.

[19] Rabini, A., Piazzini, D.B., Tancredi, G., Foti, C., Milano, G., Ronconi, G., Specchia, A., Ferrara, P.E., Maggi, L., Amabile, E., Galli, M., Bernabei, R., Bertolini, C. and Marzetti, E. (2012) Deep Heating Therapy via Microwave Dia- 
thermy Relieves Pain and Improves Physical Function in Patients with Knee Osteoarthritis: A Double-Blind Randomized Clinical Trial. European Journal of Physical and Rehabilitation Medicine, 48, 549-559.

[20] Oakley, E., Pardeiro, R.B., Powell, J.W. and Millar, A.L. (2013) The Effects of Multiple Daily Applications of Ice to the Hamstrings on Biochemical Measures, Signs, and Symptoms Associated With Exercise-Induced Muscle Damage. Journal of Strength and Conditioning Research, 27, 2743-2751. http://dx.doi.org/10.1519/JSC.0b013e31828830df 